\title{
Selection for Milk Production and Persistency Using Eigenvectors of the Random Regression Coefficient Matrix
}

\author{
K. Togashi*1 and C. Y. Lin† \\ *National Agricultural Research Center for Hokkaido Region, Hitsujigaoka 1, Toyohiraku, Sapporo, Japan 0628555 \\ †Dairy and Swine Research and Development Centre, Agriculture and Agri-Food Canada, University of Guelph, Guelph, \\ Ontario, Canada N1G 2W1
}

\section{ABSTRACT}

The purpose of this study was to investigate the relationships of the eigenvectors of the additive genetic random regression coefficient matrix $(\mathbf{K})$ to selection responses and to determine how many eigenvectors are necessary in the breeding goal to explain the variation. The construction of various eigenvector indexes was based on the $\mathbf{K}$ matrix estimated from test-day records of Japanese Holstein cattle. The first (leading) eigenvector index produced constant responses for each day of lactation, indicating that the first eigenvector is responsible for scaling the lactation curve without altering its shape. Daily genetic responses to the second eigenvector index increased linearly as DIM increased. Genetic responses to the third eigenvector index were negative in mid-lactation but were positive in early and late lactation (concave curve). Genetic responses to the fourth and fifth eigenvector indexes hovered around zero across the lactation. The results suggest that both second and third eigenvectors account for the change in the shape of the lactation curve and there is little utility of the fourth and fifth eigenvectors in improving lactation milk or persistency. When the goal is to increase lactation milk yield alone, the index based on the first eigenvector produced a similar response to the index based on all 5 eigenvectors. When the goal is to improve both lactation milk yield and persistency, the index based on the first 3 eigenvectors achieved more than $99.9 \%$ of the genetic response to an index based on all 5 eigenvectors. The advantage of an eigenvector index over conventional selection based on total lactation milk yield increases with increasing economic weight assigned to persistency.

Key words: lactation curve, lactation milk, persistency, eigenvector index

Received September 20, 2005.

Accepted July 6, 2006.

${ }^{1}$ Corresponding author: tkenji@naro.affrc.go.jp

\section{INTRODUCTION}

Lactation yield and persistency are 2 economically important traits in dairy production. Gengler (1996) reviewed various measures of lactation persistency. Togashi and Lin (2003, 2004a,b) divided the lactation into various stages to construct a "stage gain" index and compared different selection criteria for improving lactation milk and persistency using Dutch test-day milk records (Pool and Meuwissen, 2000). The stage gain index was designed to realize the intended stage gain with the least selection intensity. Lin and Togashi (2005) compared different selection strategies to maximize lactation milk without decreasing persistency.

Olori et al. (1999) reported that the first (leading) eigenvector of the additive genetic covariance function was related to increased mean milk yields at each stage of lactation; and the second eigenvector was related to the change in the shape of the lactation curve. Macciotta et al. (2004) used the eigenvectors of the correlation matrix of test-day records to derive 2 index scores, which were related to the rate of ascent to the lactation peak and the rate of decline after the peak (i.e., persistency). That study pointed out the possible relationship of the eigenvectors to lactation milk and persistency on a population basis, but did not show how to use them for genetic selection. The objectives of this paper are to 1) demonstrate the construction of various eigenvector indexes for improving lactation milk and persistency, 2) characterize the genetic response patterns across lactation of individual eigenvectors in response to selection, 3) study the selection effectiveness of eigenvector indexes as compared with conventional selection based on lactation EBV, and 4) determine how many eigenvectors are necessary to explain most of the variation in the breeding goal.

\section{MATERIALS AND METHODS}

\section{Basic Concept of Constructing an Eigenvector- Based Index}

Let $\boldsymbol{\alpha}=\left[\begin{array}{llll}\alpha_{0} & \alpha_{1} & \cdots & \alpha_{k-1}\end{array}\right]^{\prime}$ be a $(\mathrm{k} \times 1)$ vector of the additive genetic random regression (RR) coeffi- 
cients due to the fitting of a Legendre polynomial of degree $(\mathrm{k}-1)$. The variance of vector $\alpha$ is a $(\mathrm{k} \times \mathrm{k})$ additive genetic $R R$ covariance matrix $(\mathbf{K})$ with $k$ pairs of eigen values $\left(\lambda_{i}\right)$ and normalized (orthogonal) eigenvectors $\left(\boldsymbol{e}_{i}, i=1,2, \ldots, \mathrm{k}\right)$. Let $\mathbf{E}$ be a $(\mathrm{k} \times \mathrm{k})$ matrix containing these orthogonal eigenvectors as columns. The genetic covariance matrix $(\mathbf{G})$ of daily yields from DIM 5 to 305 is $\mathbf{G}=\boldsymbol{\Phi} \mathbf{K} \boldsymbol{\Phi}^{\prime}$ where $\boldsymbol{\Phi}$ is a $(301 \times \mathrm{k})$ matrix of Legendre polynomial coefficients (i.e., covariates) evaluated from DIM 5 through 305.

The index $\left(\mathrm{I}_{\mathrm{K}}\right)$ constructed based on the eigenvectors of $\mathbf{K}$ is defined as

$$
\mathrm{I}_{K}=\sum_{i=1}^{k} b_{i}\left(\boldsymbol{\alpha}^{\prime} \boldsymbol{e}_{i}\right)=\boldsymbol{b}^{\prime} \mathbf{E}^{\prime} \boldsymbol{\alpha}
$$

where $\boldsymbol{b}$ is a $(\mathrm{k} \times 1)$ vector of index coefficients. By this definition, an "index trait" $\left(\boldsymbol{\alpha}^{\prime} \boldsymbol{e}_{i}\right)$ is a linear combination of additive genetic $R R$ coefficients weighted by the elements of a given eigenvector, thus resulting in a total of $k$ index traits. The first index trait corresponds to the first eigenvector with the largest eigen value, the second index trait corresponds to the second eigenvector with the second largest eigen value, and so on. In statistical terms, these "synthetic" index traits are principal components. The variance of index $\mathrm{I}_{\mathrm{K}}$ is $\sigma_{\mathrm{I}_{\mathrm{K}}}^{2}=$ $\boldsymbol{b}^{\prime} \mathbf{E}^{\prime} \mathbf{K E} \boldsymbol{b}=\boldsymbol{b}^{\prime} \mathbf{D} \boldsymbol{b}$ where $\mathbf{D}$ is a diagonal matrix with the eigen values of $\mathbf{K}$ as the diagonal elements (Searle, 1966), indicating that the index traits are uncorrelated. The total variance of these index traits is $\operatorname{Var}\left(\mathbf{1}^{\prime} \mathbf{E}^{\prime} \boldsymbol{\alpha}\right)=\mathbf{1}^{\prime} \mathbf{D} \mathbf{1}=\sum_{i=1}^{k} \lambda_{i}$ with $\mathbf{1}$ being the summing vector of order $k$.

\section{Construction of Individual Eigenvector Indexes}

The $\mathbf{K}$ matrix used in this study was estimated using a quartic Legendre polynomial $(k=5)$ under a $R R$ test-day animal model with the first-lactation milk of Japanese Holstein cows (Togashi et al., 2005). A quartic Legendre polynomial $(k=5)$ resulted in 5 eigenvectors and thus, 5 index traits. To study the characteristics of each eigenvector of $\mathbf{K}$, the genetic response associated with each individual eigenvector was computed. Each index trait was used separately as a selection criterion $\left(\mathrm{I}_{(i \mathrm{th})}=\boldsymbol{\alpha}^{\prime} \boldsymbol{e}_{i}\right.$ where $i$ th $=$ first, second, third, fourth, or fifth) to assess the impact of the $i$ th eigenvector on lactation milk and persistency. Let $\boldsymbol{g}$ be a $(301 \times 1)$ vector of genetic values from DIM 5 to 305 . The genetic responses from DIM 5 through $305(\underline{\Delta})$ to selection on $\mathrm{I}_{(i \mathrm{th})}=\boldsymbol{\alpha}^{\prime} \boldsymbol{e}_{i}$ is as follows:

$$
\underline{\Delta}=\frac{\operatorname{Cov}\left(\boldsymbol{g}, \boldsymbol{I}_{i}\right)}{\sigma_{I_{(i \mathrm{th})}}^{2}}(S D)=\frac{\operatorname{Cov}\left(\boldsymbol{\Phi} \boldsymbol{\alpha}, \boldsymbol{\alpha}^{\prime} \boldsymbol{e}_{i}\right)}{\sigma_{I_{(i \mathrm{th})}}^{2}}(\mathrm{SD})=\boldsymbol{\Phi} \mathbf{K} \boldsymbol{e}_{i}\left(\frac{\bar{i}}{\sigma_{I_{(i \mathrm{th})}}}\right)
$$

where $\Delta=\left[\begin{array}{llll}\Delta G_{5} & \Delta G_{6} & \cdots & \Delta G_{305}\end{array}\right]^{\prime}$, SD is selection differential, $\bar{i}$ is selection intensity, and $\sigma_{\mathrm{I}_{(i t h)}^{2}}^{2}=\mathbf{e}_{i}^{\prime} \mathbf{K} \mathbf{e}_{i}=\lambda_{i}$.

\section{Sequential Eigenvector Indexes for Improving Lactation Milk}

Let the breeding goal be to improve the genetic value of lactation milk $\left(G_{L}=\mathbf{1}^{\prime} \boldsymbol{\Phi} \boldsymbol{\alpha}\right)$. Then, the eigenvector index I can be derived as follows:

$$
\begin{gathered}
f=\operatorname{Var}\left(\mathrm{I}-G_{L}\right)=\operatorname{Var}\left(\boldsymbol{b}^{\prime} \mathbf{E}^{\prime} \boldsymbol{\alpha}-\mathbf{1}^{\prime} \boldsymbol{\Phi} \boldsymbol{\alpha}\right) \\
=\boldsymbol{b}^{\prime} \mathbf{E}^{\prime} \mathbf{K} \mathbf{E} \boldsymbol{b}+\mathbf{1}^{\prime} \boldsymbol{\Phi} \mathbf{K} \boldsymbol{\Phi}^{\prime} \mathbf{1}-2 \boldsymbol{b}^{\prime} \mathbf{E}^{\prime} \mathbf{K} \boldsymbol{\Phi}^{\prime} \mathbf{1} \\
=\boldsymbol{b}^{\prime} \mathbf{D} \boldsymbol{b}+\mathbf{1}^{\prime} \mathbf{G} \mathbf{1}-2 \boldsymbol{b}^{\prime} \mathbf{E}^{\prime} \mathbf{K} \boldsymbol{\Phi}^{\prime} \mathbf{1}
\end{gathered}
$$

Differentiating the function $f$ with respect to $\boldsymbol{b}$ and setting the resulting derivatives equal to zero lead to the following:

$$
\frac{\partial f}{\partial \boldsymbol{b}}=2 \mathbf{D} \boldsymbol{b}-2 \mathbf{E}^{\prime} \mathbf{K} \boldsymbol{\Phi}^{\prime} \mathbf{1}=0 .
$$

Thus,

$$
\boldsymbol{b}=\mathbf{D}^{-1} \mathbf{E}^{\prime} \mathbf{K} \Phi^{\prime} \mathbf{1}
$$

The genetic responses $(\underline{\Delta})$ of daily yields from DIM 5 to 305 are

$$
\underline{\Delta}=\frac{\operatorname{Cov}\left(\boldsymbol{\Phi} \boldsymbol{\alpha}, \boldsymbol{b}^{\prime} \mathbf{E}^{\prime} \boldsymbol{\alpha}\right)}{\sigma_{\mathrm{I}}^{2}}(S D)=\boldsymbol{\Phi} \mathbf{K E} \boldsymbol{b}\left(\bar{i} / \sigma_{\mathrm{I}}\right)
$$

where $\sigma_{\mathrm{I}}^{2}=\boldsymbol{b}^{\prime} \mathbf{D} \boldsymbol{b}$.

The full eigenvector index denoted by $\mathrm{I}_{(5)}$ consists of 5 index traits. The last index trait of the full eigenvector index was dropped sequentially to yield 4 reduced indexes $I_{(4)}, I_{(3)}, I_{(2)}$, and $I_{(1)}$ where the number in parenthesis indicates the number of index traits included in an index. For example, the last index trait of $\mathrm{I}_{(5)}$ was dropped to yield $\mathrm{I}_{(4)}$; the last index trait of $\mathrm{I}_{(4)}$ was dropped to produce $\mathrm{I}_{(3)}$; and so on. The full and reduced indexes were computed according to [1]. Because the index traits are orthogonal, the index coefficient for a given index trait is the same among these sequential indexes (e.g., $b_{2}$ for the second index trait in $I_{(2)}, I_{(3)}$, $I_{(4)}$, and $I_{(5)}$ are identical). Genetic response to each of these sequential indexes was computed based on [2]. 


\section{Sequential Eigenvector Index for Improving Lactation Milk and Persistency}

Let $g_{280}$ and $g_{55}$ be the genetic values at DIM 280 and 55 , respectively, and $\mathrm{a}_{1}$ and $\mathrm{a}_{2}$ be the economic weights of lactation milk and persistency. The eigenvector index (I*) designed to maximize a linear combination of lactation milk and persistency is defined as

$$
I^{*}=\boldsymbol{b}^{\prime} \mathbf{E}^{\prime} \alpha,
$$

and net merit:

$$
H=a_{1} \mathbf{1}^{\prime} \mathbf{g}^{*}+a_{2}\left(g_{280}-g_{55}\right)
$$

where $\mathbf{g}^{*}$ is a $(299 \times 1)$ vector of genetic values from DIM 5 to 305 excluding $g_{280}$ and $g_{55}$. The exclusion of $g_{280}$ and $g_{55}$ from $\mathbf{g}^{*}$ is to avoid duplication because the second trait of the net merit $(\mathrm{H})$ contains both $g_{280}$ and $g_{55}$. The measure of persistency is defined as the difference between $g_{280}$ and $g_{55}$. Togashi and Lin (2004b) compared the efficiency of 5 different selection criteria for persistency and found that selection on the difference in EBV between the peak and DIM 280 achieved the greatest persistency. First-lactation milk of the Japanese Holstein cows peaked at DIM 55 (Togashi et al., 2005). The eigenvector index designed to maximize net merit is derived as follows:

$$
\begin{gathered}
f=\mathrm{E}\left(\mathrm{I}^{*}-\mathrm{H}\right)^{2} \\
=\boldsymbol{b}^{\prime} \mathbf{D} \boldsymbol{b}+a_{1}^{2} \mathbf{1}^{\prime} \mathbf{G}^{*} \mathbf{1}+a_{2}^{2} \sigma_{g_{280}}^{2} g_{55} \\
+2 a_{1} a_{2} \mathbf{1}^{\prime}\left(\mathbf{G}_{280}-\mathbf{G}_{55}\right) \\
-2 \boldsymbol{b}^{\prime} \mathbf{E}^{\prime} \mathbf{K}\left(a_{1} \boldsymbol{\Phi}^{* \prime} \mathbf{1}+a_{2}\left(\boldsymbol{\Phi}_{280}^{\prime}-\boldsymbol{\Phi}_{55}^{\prime}\right)\right)
\end{gathered}
$$

where $\mathbf{G}^{*}$ is a $(299 \times 299)$ submatrix of $\mathbf{G}$ by deleting the rows and columns of $\mathbf{G}$ corresponding to DIM 55 and 280; vectors $\mathbf{G}_{55}$ and $\mathbf{G}_{280}$ refer to the columns of $\mathbf{G}$ corresponding to DIM 55 and 280 , respectively; $\boldsymbol{\Phi}_{55}$ and $\boldsymbol{\Phi}_{280}$ refer to the rows of $\boldsymbol{\Phi}$ corresponding to DIM 55 and 280 , respectively; $\Phi^{*}$ is a $(299 \times \mathrm{k})$ matrix obtained by deleting the rows of $\Phi$ corresponding to DIM 55 and 280; and $a_{1}$ and $a_{2}$ are economic weights between lactation milk and persistency. Because the relative economic weights between lactation milk and persistency were not available in literature, their economic weights were assumed to be $a_{1}: a_{2}=1: 50$ and $a_{1}: a_{2}=1: 100$. Taking the derivative of the function $f$ with respect to $\boldsymbol{b}$ and setting the partial derivatives to zero result in the following equations:

$$
\frac{\partial f}{\partial \boldsymbol{b}}=2 \mathbf{D} \boldsymbol{b}-2 \mathbf{E}^{\prime} \mathbf{K}\left(a_{1} \boldsymbol{\Phi}^{* \prime} \mathbf{1}^{\prime}+a_{2}\left(\boldsymbol{\Phi}_{280}^{\prime}-\boldsymbol{\Phi}_{55}^{\prime}\right)\right)=0 .
$$

Thus, the index for maximizing the response in net merit of lactation milk and persistency is,

$$
\boldsymbol{b}=\mathbf{D}^{-1} \mathbf{E}^{\prime} \mathbf{K}\left(a_{1} \boldsymbol{\Phi}^{* \prime} \mathbf{1}+a_{2}\left(\boldsymbol{\Phi}_{280}^{\prime}-\boldsymbol{\Phi}_{55}^{\prime}\right)\right)
$$

The genetic responses $(\underline{\Delta})$ of daily yields from DIM 5 to 305 are computed as follows:

$$
\begin{gathered}
\underline{\Delta}=\boldsymbol{\Phi K E} \mathbf{b}\left(\bar{i} / \sigma_{I^{*}}\right) \\
\sigma_{I^{*}}^{2}=\boldsymbol{b}^{\prime} \mathbf{E}^{\prime} \mathbf{K E} \boldsymbol{b}=\boldsymbol{b}^{\prime} \mathbf{D} \boldsymbol{b} .
\end{gathered}
$$

The fitting of quartic Legendre polynomial $(k=5)$ results in a full index, $I_{(5)}^{*}$, that consists of 5 index traits resulting from the 5 eigenvectors. Dropping the last index trait sequentially produced the reduced indexes $I_{(4)}^{*}, I_{(3)}^{*}$, $I_{(2)}^{*}$, and $I_{(1)}^{*}$. Genetic responses to this series of sequential indexes were computed according to [4]. Selection intensity is set at 1.0 for all selection criteria compared in this study. The genetic responses to different indexes compared were computed and used for direct comparisons.

\section{Numerical Example for Constructing Eigenvector Index}

The construction of an eigenvector index for improving lactation milk and persistency with $\mathrm{a}_{1}: \mathrm{a}_{2}=1: 50$ may be illustrated. The additive genetic $R R$ covariance matrix (K) was estimated using a quartic Legendre polynomial $(k=5)$ under a test-day animal model (Togashi et al., 2005):

$$
\mathbf{K}=\left[\begin{array}{rrrrr}
12.244 & 0.757 & -0.912 & 0.424 & -0.131 \\
0.757 & 0.997 & -0.170 & -0.001 & 0.029 \\
-0.912 & -0.170 & 0.308 & -0.029 & 0.020 \\
0.424 & -0.001 & -0.029 & 0.076 & -0.020 \\
-0.131 & 0.029 & 0.020 & -0.020 & 0.049
\end{array}\right]
$$

Eigen values and eigenvectors of matrix $\mathrm{K}$ were shown in Table 1. It follows that

$$
\mathbf{E}=\left[\begin{array}{rrrrr}
0.9942 & -0.0762 & 0.0667 & -0.0349 & -0.0117 \\
0.0672 & 0.9849 & 0.1472 & 0.0541 & -0.0279 \\
-0.0762 & -0.1463 & 0.9829 & 0.0503 & -0.0652 \\
0.0344 & -0.0332 & -0.0122 & 0.8580 & 0.5112 \\
-0.0106 & 0.0397 & 0.0879 & -0.5071 & 0.8564
\end{array}\right]
$$


Table 1. Eigenvalues (with proportion of total variation explained ${ }^{1}$ ) and eigenvectors of the additive genetic random regression coefficient matrix $(\mathbf{K})$ with quartic Legendre polynomials

\begin{tabular}{|c|c|c|c|c|c|}
\hline & \multicolumn{5}{|c|}{ Eigenvalue $\left(\lambda_{i}\right)$} \\
\hline & 1 & 2 & 3 & 4 & 5 \\
\hline & $\begin{array}{l}12.381 \\
(0.9054)\end{array}$ & $\begin{array}{c}0.965 \\
(0.0706)\end{array}$ & $\begin{array}{l}0.223 \\
(0.0163)\end{array}$ & $\begin{array}{l}0.069 \\
(0.0051)\end{array}$ & $\begin{array}{c}0.036 \\
(0.0027)\end{array}$ \\
\hline \multicolumn{6}{|l|}{ Eigenvectors $\left(\boldsymbol{e}_{i}\right)$} \\
\hline Constant & 0.9942 & -0.0762 & 0.0667 & -0.0349 & -0.0117 \\
\hline 1 (Linear) & 0.0672 & 0.9849 & 0.1472 & 0.0541 & -0.0279 \\
\hline 2 (Quadratic) & -0.0762 & -0.1463 & 0.9829 & 0.0503 & -0.0652 \\
\hline 3 (Cubic) & 0.0344 & -0.0332 & -0.0122 & 0.8580 & 0.5112 \\
\hline 4 (Quartic) & -0.0106 & 0.0397 & 0.0879 & -0.5071 & 0.8564 \\
\hline
\end{tabular}

and $\mathbf{E}^{\prime} \mathbf{K E}=\mathbf{D}=\left[\begin{array}{ccccc}12.381 & 0 & 0 & 0 & 0 \\ 0 & 0.965 & 0 & 0 & 0 \\ 0 & 0 & 0.223 & 0 & 0 \\ 0 & 0 & 0 & 0.069 & 0 \\ 0 & 0 & 0 & 0 & 0.036\end{array}\right]$

$\Phi$ is a $(301 \times 5)$ matrix of Legendre polynomial coefficients evaluated from DIM 5 to 305 ;

$$
\Phi=\left[\begin{array}{ccccc}
0.707 & -1.225 & 1.581 & -1.871 & 2.121 \\
0.707 & -1.217 & 1.550 & -1.797 & 1.982 \\
\vdots & \vdots & \vdots & \vdots & \vdots \\
0.707 & 1.225 & 1.581 & 1.871 & 2.121
\end{array}\right]
$$

$\Phi_{55}$ and $\boldsymbol{\Phi}_{280}$ refer to the rows of $\boldsymbol{\Phi}$ corresponding to DIM 55 and 280, respectively:

$$
\begin{aligned}
& \Phi_{55}=\left(\begin{array}{lllll}
0.707 & -0.816 & 0.264 & 0.485 & -0.907
\end{array}\right) \\
& \Phi_{280}=\left(\begin{array}{lllll}
0.707 & 1.021 & 0.856 & 0.368 & -0.253
\end{array}\right) ;
\end{aligned}
$$

and $\Phi^{*}$ is a $(299 \times 5)$ matrix formed by deleting $\boldsymbol{\Phi}_{55}$ and $\boldsymbol{\Phi}_{280}$ from $\boldsymbol{\Phi}$.

Substituting the relevant matrix elements into [3] gives the following index coefficients,

$$
\mathbf{b}=(213.45,71.40,60.44,-24.91,20.40)^{\prime}
$$

Therefore, $\mathrm{I}^{*}=213.45 \mathrm{x}_{1}+71.40 \mathrm{x}_{2}+60.44 \mathrm{x}_{3}-$ $24.91 \mathrm{x}_{4}+20.40 \mathrm{x}_{5}$

where $\mathrm{x}_{1}=\mathbf{e}_{1}^{\prime} \boldsymbol{\alpha}, \mathrm{x}_{2}=\mathbf{e}_{2}^{\prime} \boldsymbol{\alpha}, \mathrm{x}_{3}=\mathbf{e}_{3}^{\prime} \alpha, \mathrm{x}_{4}=\mathbf{e}_{4}^{\prime} \boldsymbol{\alpha}$, and $\mathrm{x}_{5}=$

$\mathbf{e}_{5}^{\prime} \boldsymbol{\alpha}$ with $\mathbf{e}_{\mathrm{i}}$ being the ith eigenvector of $\mathbf{K}$ and $\boldsymbol{\alpha}=$ $\left(\begin{array}{lllll}\alpha_{0} & \alpha_{1} & \alpha_{2} & \alpha_{3} & \alpha_{4}\end{array}\right)^{\prime}$ being the additive genetic RR coefficients of each animal.

\section{RESULTS AND DISCUSSION}

\section{Genetic Responses to Selection Based on Individual Eigenvectors}

The eigen values and eigenvectors of the additive genetic RR coefficient matrix (K) with quartic Legendre polynomials were shown in Table 1 . The first 3 eigen values explained $90.54,7.06$, and $1.63 \%$ of the variation of the random regression coefficients, respectively, whereas the remaining 2 eigen values accounted for a combined total of $0.78 \%$. The first element in the leading eigenvector is the largest, suggesting that the first eigenvector contributes mainly to the variation of the constant throughout the lactation. The remaining 4 eigenvectors have the largest elements associated with linear, quadratic, cubic, and quartic parts of the curve, respectively, and affect the fluctuation of the lactation curve in various ways.

Genetic responses in lactation milk and persistency to the 5 individual eigenvector indexes $\left(\mathrm{I}_{\text {(first) }}, \mathrm{I}_{\text {(second) }}\right.$, $\mathrm{I}_{\text {(third) }}, \mathrm{I}_{\text {(fourth) }}$, and $\mathrm{I}_{\text {(fifth) }}$ ) were compared with conventional selection on lactation milk in Table 2. Genetic responses to the first eigenvector index $\left(\mathrm{I}_{(\mathrm{first})}\right)$ and to selection on lactation milk $\left(\mathrm{I}_{\mathrm{G}_{\mathrm{L}}}\right)$ were similar. The sec-

Table 2. Genetic responses (kg) to individual eigenvector indexes in comparison with conventional selection on lactation milk yield $\left(I_{G_{L}}\right)$

\begin{tabular}{lrrrr}
\hline Criterion $^{1}$ & $\Delta \mathrm{G}_{L}{ }^{2}$ & $\Delta \mathrm{G}_{P}{ }^{3}$ & $\Delta \mathrm{G}_{55}$ & $\Delta \mathrm{G}_{280}$ \\
\hline $\mathrm{I}_{(1 \text { st }}$ & 744.1 & 0.237 & 2.30 & 2.54 \\
$\mathrm{I}_{(2 \text { nd })}$ & -16.1 & 1.722 & -0.93 & 0.79 \\
$\mathrm{I}_{(3 \mathrm{rd})}$ & 7.5 & 0.431 & 0.05 & 0.48 \\
$\mathrm{I}_{(4 \text { th })}$ & -2.2 & -0.080 & 0.22 & 0.14 \\
$\mathrm{I}_{(5 \text { th })}$ & -0.1 & 0.078 & -0.10 & -0.02 \\
$I_{G_{L}}$ & 744.3 & 0.204 & 2.32 & 2.53 \\
\hline
\end{tabular}

${ }^{1} \mathrm{I}_{(i)}$ is the eigenvector index constructed from the $i$ th eigenvector of the additive genetic random regression coefficient matrix $\mathbf{K}$.

${ }^{2} \Delta \mathrm{G}_{L}=$ Genetic gain in lactation milk.

${ }^{3} \Delta \mathrm{G}_{P}=\Delta \mathrm{G}_{280}-\Delta \mathrm{G}_{55}$. 


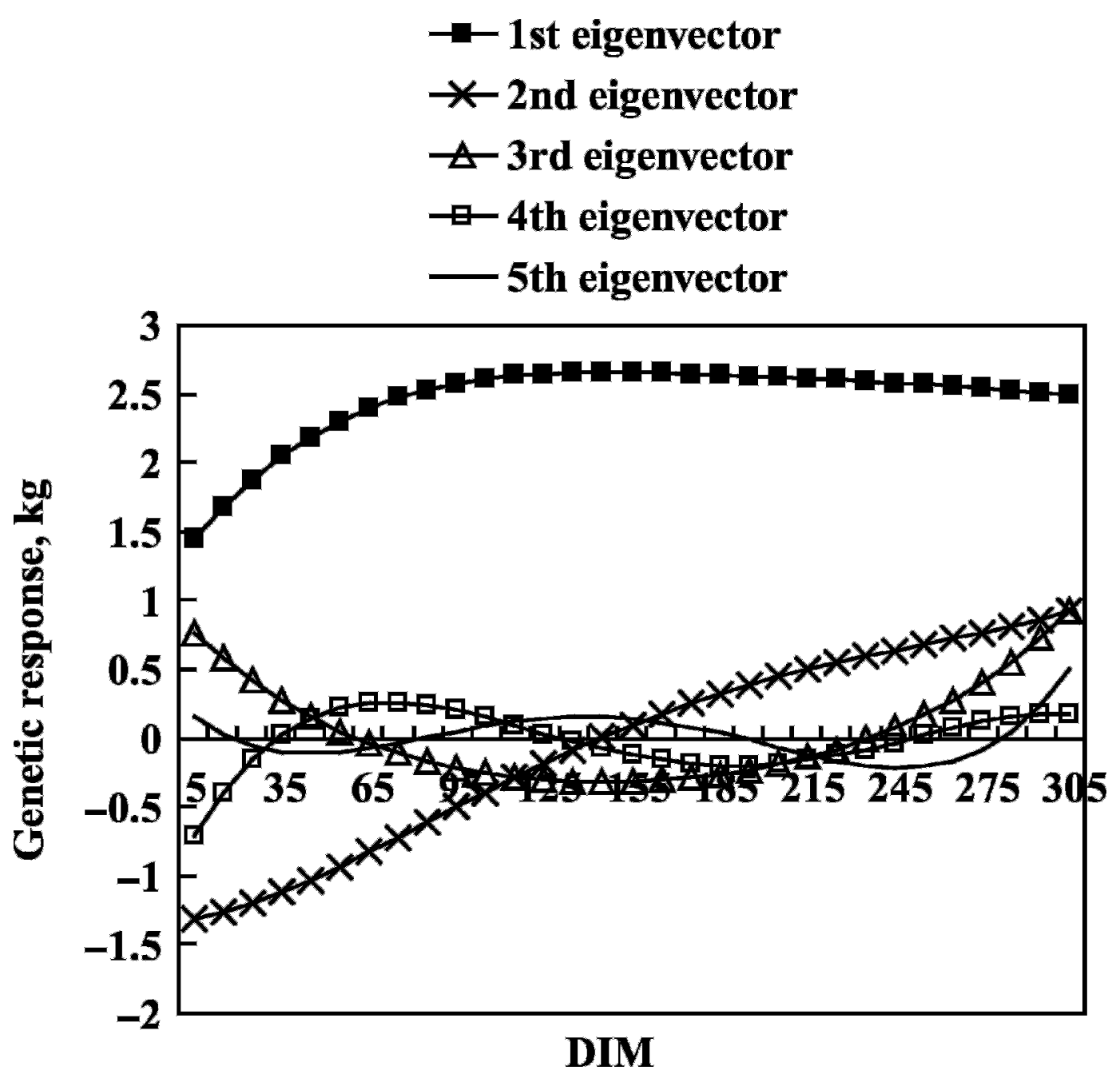

Figure 1. Genetic responses across lactation due to selection based on individual eigenvectors.

ond eigenvector index decreased both lactation milk and peak yield but increased milk at DIM 280, thus increasing persistency. The third eigenvector index slightly increased both lactation milk and persistency whereas the fourth eigenvector index decreased both lactation milk and persistency. The genetic responses to the fifth eigenvector index in lactation milk and persistency were negligible.

The patterns of daily genetic responses to the 5 individual eigenvector indexes from DIM 5 to 305 are in Figure 1. The genetic responses in daily milk to the first eigenvector index increased from $1.5 \mathrm{~kg}$ at DIM 5 to $2.6 \mathrm{~kg}$ at DIM 69 and then stabilized at $2.5 \mathrm{~kg}$ from DIM 70 to 305 . The horizontal pattern of the first eigenvector index indicates that the first eigenvector accounts mainly for constant increase in daily milk across lactation. Thus, the first eigenvector index $\mathrm{I}_{\text {(first) }}$ is responsible for raising the level of milk production without changing the shape of the lactation curve. Genetic responses to the second eigenvector index were negative before DIM 143 and positive thereafter, showing a positive, linear relationship between daily genetic response and DIM. The second eigenvector index achieved a greater persistency than any other individual eigenvector indexes or selection on lactation milk, but was coupled with a loss of $16.1 \mathrm{~kg}$ in lactation milk (Table 2). These results suggest that the second eigenvector was highly related to the change of the lactation curve.

The daily genetic responses to the third eigenvector index were positive before DIM 60, negative between DIM 61 and 237, and positive from DIM 238 to the end of the lactation, forming a concave curve (Figure 1). The third eigenvector index achieved a genetic gain of $7.5 \mathrm{~kg}$ in lactation milk and produced the second largest persistency next to the second eigenvector in- 
Table 3. Genetic responses $(\mathrm{kg})$ to sequential eigenvector indexes when net merit consists of lactation milk alone or a linear combination of lactation milk and persistency

\begin{tabular}{|c|c|c|c|c|c|}
\hline Criteria $^{1}$ & $\Delta \mathrm{G}_{L}$ & $\Delta \mathrm{G}_{P}{ }^{2}$ & $\Delta \mathrm{G}_{55}$ & $\Delta \mathrm{G}_{280}$ & $\Delta \mathrm{H}^{3}$ \\
\hline \multicolumn{6}{|l|}{ Selection for lactation milk alone } \\
\hline$I_{(1)}=211.45 x_{1}$ & 744.1 & 0.237 & 2.30 & 2.54 & \\
\hline $\mathrm{I}_{(2)}=211.45 x_{1}-16.37 x_{2}$ & 744.2 & 0.200 & 2.32 & 2.52 & \\
\hline$I_{(3)}=211.45 x_{1}-16.37 x_{2}+15.95 x_{3}$ & 744.3 & 0.204 & 2.32 & 2.53 & \\
\hline$I_{(4)}=211.45 x_{1}-16.37 x_{2}+15.95 x_{3}-8.44 x_{4}$ & 744.3 & 0.205 & 2.32 & 2.53 & \\
\hline$I_{(5)}=211.45 x_{1}-16.37 x_{2}+15.95 x_{3}-8.44 x_{4}-0.76 x_{5}$ & 744.3 & 0.204 & 2.32 & 2.53 & \\
\hline \multicolumn{6}{|l|}{ Selection for lactation milk and persistency with $a_{1}: a_{2}=1: 50^{4}$} \\
\hline$I_{(1)}^{*}=213.45 x_{1}$ & 744.1 & 0.237 & 2.30 & 2.54 & 751.1 \\
\hline $\mathrm{I}_{(2)}^{*}=213.45 x_{1}+71.40 x_{2}$ & 739.3 & 0.396 & 2.21 & 2.60 & 754.3 \\
\hline$I_{(3)}^{*}=213.45 x_{1}+71.40 x_{2}+60.44 x_{3}$ & 739.1 & 0.412 & 2.21 & 2.62 & 754.9 \\
\hline$I_{(4)}^{*}=213.45 x_{1}+71.40 x_{2}+60.44 x_{3}-24.91 x_{4}$ & 739.1 & 0.413 & 2.20 & 2.62 & 754.9 \\
\hline $\mathrm{I}_{(5)}^{*}=213.45 x_{1}++71.40 x_{2}+60.44 x_{3}-24.91 x_{4}+20.40 x_{5}$ & 739.1 & 0.413 & 2.20 & 2.62 & 754.9 \\
\hline $\mathrm{I}_{\mathrm{G}_{\mathrm{L}}}$ & 744.3 & 0.204 & 2.32 & 2.53 & 749.7 \\
\hline \multicolumn{6}{|l|}{ Selection for lactation milk and persistency with $a_{1}: a_{2}=1: 100$} \\
\hline$I_{(1)}^{*}=216.82 x_{1}$ & 744.1 & 0.237 & 2.30 & 2.54 & 763.0 \\
\hline $\mathrm{I}_{(2)}^{*}=216.82 x_{1}+159.02 x_{2}$ & 725.7 & 0.578 & 2.07 & 2.65 & 778.8 \\
\hline$I_{(3)}^{*}=216.82 x_{1}+159.02 x_{2}+106.04 x_{3}$ & 724.7 & 0.604 & 2.07 & 2.67 & 780.4 \\
\hline$I_{(4)}^{*}=216.82 x_{1}+159.02 x_{2}+106.04 x_{3}-40.04 x_{4}$ & 724.7 & 0.605 & 2.06 & 2.67 & 780.5 \\
\hline$I_{(5)}^{*}=216.82 x_{1}+159.02 x_{2}+106.04 x_{3}-40.04 x_{4}+40.91 x_{5}$ & 724.6 & 0.606 & 2.06 & 2.67 & 780.5 \\
\hline $\mathrm{I}_{\mathrm{G}_{\mathrm{L}}}$ & 744.3 & 0.204 & 2.32 & 2.53 & 759.9 \\
\hline
\end{tabular}

${ }^{1}$ Eigenvector index $\mathrm{I}_{(n)}$ where subscript $n$ indicates the first $n$ index traits used for index construction and $x_{i}$ is the $i$ th index trait.

${ }^{2} \Delta \mathrm{G}_{P}=\Delta \mathrm{G}_{280}-\Delta \mathrm{G}_{55}$.

${ }^{3} \Delta \mathrm{H}=a_{1} \Delta g^{*}+a_{2} \Delta\left(g_{280}-g_{55}\right), \Delta \mathrm{g}^{*}=$ genetic gain from DIM 5 through 305 excluding $\mathrm{g}_{280}$ and $\mathrm{g}_{55}$.

${ }^{4} a_{1}$ and $a_{2}$ are economic weights between lactation milk and persistency.

dex (Table 2). Therefore, the third eigenvector has a curvilinear effect on the shape of the lactation curve. Further study on how to flatten the concave curve to increase milk yield and persistency is warranted.

Genetic responses in daily milk to the fourth and the fifth eigenvector indexes hovered around zero across lactation (Figure 1), giving a combined gain of -2.3 $\mathrm{kg}$ in lactation milk. Furthermore, these 2 individual eigenvector indexes produced little response in persistency (Table 2). The combined results indicate that both the fourth and fifth eigenvectors play a minimal role in genetic improvement of lactation milk and persistency, indicating that the fourth and fifth eigenvectors can be excluded from the analysis. Note that Legendre polynomial terms 4 and 5 do not correspond to eigenvectors 4 and 5 . Therefore, it makes no difference in fitting quadratic $(\mathrm{k}=3)$ or quartic $(\mathrm{k}=5)$ Legendre polynomials to Japanese test-day data. Obviously, the use of a quadratic rather than a quartic Legendre polynomial would greatly reduce computational requirements under a test-day animal model. In this regard, Druet et al. (2003) pointed out that the model using the eigenvectors of the additive genetic covariance matrix would reduce the computational requirements and improve its convergence properties. The degree of the additive genetic RR Legendre polynomial fitted to a given data set should be determined not only by computational costs but genetic responses as well. It merits further study to utilize the genetic response pattern of individual eigenvectors to modify the shape of the lactation curve.

\section{Sequential Eigenvector Indexes Designed to Maximize Lactation Milk}

Genetic responses $(\mathrm{kg})$ to a series of sequential eigenvector indexes designed to maximize lactation milk are in Table 3. Genetic responses to each of the sequential eigenvector indexes constructed by dropping one eigenvector at a time $\left(\mathrm{I}_{(5)}, \mathrm{I}_{(4)}, \mathrm{I}_{(3)}, \mathrm{I}_{(2)}, \mathrm{I}_{(1)}\right)$ are almost the same, suggesting that extra genetic gains by adding extra eigenvectors to the first (leading) eigenvector index are minimal when the breeding goal is to improve lactation milk alone. Index coefficient of 211.45 for the first eigenvector is much larger than any other 4 eigenvectors (Table 3 ). Because the index traits are orthogonal, the genetic responses in the 5 index traits are additive and the variance of $I_{(5)}$ is equal to the sum of the variances for the 5 individual indexes. 


\section{Sequential Eigenvector Indexes Designed to Maximize Lactation Milk and Persistency}

Genetic responses $(\mathrm{kg})$ to the eigenvector index I* designed to improve lactation milk and persistency were shown in Table 3 . When economic weights between lactation milk and persistency were assumed to be $a_{1}: a_{2}=1: 50$, index $\mathrm{I}_{(5)}^{*}$ decreased lactation milk by $5.2 \mathrm{~kg}$ of EBV but doubled persistency as compared with selection based on lactation EBV (Table 3). When $a_{1}: a_{2}=1: 100$, index $I_{(5)}^{*}$ decreased lactation milk by $19.7 \mathrm{~kg}$ of EBV but tripled persistency as compared with selection on lactation EBV (Table 3). When the goal was to maximize lactation milk alone, index coefficient for the second eigenvector $\left(b_{2}\right)$ was -16.4 . In contrast, when the goal was to maximize lactation milk and persistency, $b_{2}$ increased to 71.4 if $a_{1}: a_{2}=1: 50$ and jumped to 159.0 if $a_{1}: a_{2}=1: 100$. The index coefficient for the third eigenvector $\left(b_{3}\right)$ was 16.0 when selection was for lactation milk alone but increased to 60.4 and 106.0 when selection was for lactation milk and persistency with $a_{1}: a_{2}=1: 50$ and $a_{1}: a_{2}=1: 100$, respectively. Thus, index coefficients associated with both the second and third eigenvectors increased as the economic weight for persistency $\left(\mathrm{a}_{2}\right)$ increased, reconfirming that the second and third eigenvectors are responsible for persistency. When $a_{1}: a_{2}=1: 50$, genetic response in net merit $(\Delta \mathrm{H})$ to selection on $\mathrm{I}_{(5)}^{*}$ was 754.9 as compared with 749.7 for conventional selection on lactation $\mathrm{EBV}\left(\mathrm{I}_{\mathrm{G}_{\mathrm{L}}}\right)$. When $a_{1}: a_{2}=1: 100$, selection on $\mathrm{I}_{(5)}^{*}$ yielded $\Delta \mathrm{H}=780.5$ vs. 759.9 for selection on $\mathrm{I}_{\mathrm{G}_{\mathrm{L}}}$. Therefore, the advantage of $\mathrm{I}_{(5)}^{*}$ over $\mathrm{I}_{\mathrm{G}_{\mathrm{L}}}$ increases as the economic weight for persistency increases. Table 3 shows that $\Delta \mathrm{H}$ increased as the number of eigenvectors used for index construction increased and approached the maximum when the first 3 eigenvectors were used to derive the index. The use of the first 2 eigenvectors explained $99.78 \%$ of the variation in the breeding goal of improving the lactation milk and persistency as opposed to $99.99 \%$ for the first 3 eigenvectors, suggesting that the use of the first 2 eigenvectors is sufficient. Although the contribution of the third eigenvector is small in this case, the importance of the third eigenvector increases with increasing economic weight on persistency; thus, it is worthwhile using the first 3 eigenvectors for the purpose of general application. The joint contribution of the fourth and fifth eigenvectors to the breeding goal is negligible. In this regard, Druet et al. (2003) suggested that using the reduced set of eigenvectors for genetic evaluation would reduce computational cost. Van der Werf et al. (1998) used the eigen values and eigenvectors to perform canonical transformation to reduce the number of RR coefficients needed and the number of equations in the mixed model equations for computational efficiency.

\section{CONCLUSIONS}

The eigenvectors of the additive genetic RR coefficient matrix were used to construct various indexes to assess their impacts on lactation milk and persistency. Genetic responses in daily milk to the first (leading) eigenvector index were constant across lactation, indicating that the first eigenvector is responsible for raising the lactation curve without changing the lactation shape. Genetic responses in daily milk to the second eigenvector index increased consistently from DIM 5 to 305 , exhibiting a positive linear relationship between genetic response and DIM. The third eigenvector index decreased daily milk in mid-lactation, but increased daily milk in early and late lactation (concave curve), leading to a small gain $(7.5 \mathrm{~kg})$ in total milk. The fourth and fifth eigenvector indexes hovered around zero across lactation, resulting in genetic gains of -2.2 and -0.1 in total milk, respectively. These results suggest that the second and third eigenvectors account for the shape of the lactation curve and the fourth and fifth eigenvectors have little use in improving lactation milk, persistency, or both. When the breeding goal is to maximize lactation milk alone, the index based on the first (leading) eigenvector produced a similar response to the full index based on 5 eigenvectors, suggesting that the second to the fifth eigenvectors provide no useful information for the improvement of total milk. However, when the breeding goal is to maximize the combined performance of lactation milk and persistency, the first 3 eigenvectors are needed to construct the desired index because inclusion of the second and third eigenvector in the index greatly increased the genetic responses in persistency. The response in $\Delta \mathrm{H}$ due to the inclusion of the second and third eigenvector in the index increases with increasing economic weight for persistency in relation to lactation milk. The first 3 eigenvectors are necessary to construct an index to maximize $\Delta \mathrm{H}$ and the fourth and the fifth eigenvectors are negligible. This study demonstrates the construction of various eigenvector indexes based on a firstparity RR test-day model, but the same principle is applicable to a multiple-lactation, test-day model.

\section{ACKNOWLEDGMENTS}

The authors are grateful to an anonymous reviewer for his helpful comments in improving the manuscript. 


\section{REFERENCES}

Druet, T., F. Jaffrézic, D. Boichard, and V. Ducrocq. 2003. Modeling lactation curves and estimation of genetic parameters for first lactation test-day records of French Holstein cows. J. Dairy Sci. 86:2480-2490.

Gengler, N. 1996. Persistency of lactation yields: A review. Interbull Bull. 12:87-96.

Lin, C. Y., and K. Togashi. 2005. Maximization of lactation milk production without decreasing persistency. J. Dairy Sci. 88:2975-2980.

Macciotta, N. P. P., D. Vicario, C. D. Mauro, and A. Cappio-Borlina. 2004. A multivariate approach to modeling shapes of individual lactation curves in cattle. J. Dairy Sci. 87:1092-1098.

Olori, V. E., W. G. Hill, B. J. McGuirk, and S. Brotherstone. 1999. Estimating variance components for test-day milk records by restricted maximum likelihood with a random regression animal model. Livest. Prod. Sci. 61:53-63.

Pool, M. H., and T. H. E. Meuwissen. 2000. Reduction of the number of parameters needed for a polynomial random regression test day model. Livest. Prod. Sci. 64:133-145.
Searle, S. R. 1966. Matrix Algebra for the Biological Science. John Wiley \& Sons, Inc., New York, NY.

Togashi, K., Y. Atagi, J. Sato, T. Shirai, H. Takeda, and T. Yamazaki. 2005. Index selection for increased milk yield in mid and late lactation with the highest probability. Sustain. Livest. Prod. Hum. Welf. 59:877-881.

Togashi, K., and C. Y. Lin. 2003. Modifying the lactation curve to improve lactation milk and persistency. J. Dairy Sci. 86:1487-1493.

Togashi, K., and C. Y. Lin. 2004a. Development of an optimal index to improve lactation yield and persistency with the least selection intensity. J. Dairy Sci. 87:3047-3052.

Togashi, K., and C. Y. Lin. 2004b. Efficiency of different selection criteria for persistency and lactation milk yield. J. Dairy Sci. $87: 1528-1535$.

Van der Werf, J. H. J., M. E. Goddard, and K. Meyer. 1998. The use of covariance functions and random regressions for genetic evaluation of milk production based on test day records. J. Dairy Sci. 81:3300-3308. 